\title{
The Effects of Polyadenylation Status on MPFs During In Vitro Porcine Oocyte Maturation
}

\author{
Huiyu Liu Yan Gao $^{\mathrm{a}}$ Bo Zhai ${ }^{\mathrm{b}}$ Hao Jiang ${ }^{\mathrm{a}}$ Yu Ding ${ }^{\mathrm{a}}$ Lianjiang Zhang \\ Changhong Lid Qiong Deng ${ }^{a}$ Xianfeng Yu Jiabao Zhanga,e
}

aLaboratory Animal Center, College of Animal Sciences, Jilin University, Changchun, ${ }^{b} B r a n c h$ of Animal Husbandry, Jilin Academy of Agricultural Science, Gongzhuling, 'College of Animal Science and Technology, Jilin Agricultural and Technology College, Jilin, 'College of Life Sciences, Bai Cheng Normal University, Baicheng, eState and Local Joint Engineering Laboratory for Animal Models of Human

Diseases, Jilin University, Changchun, China

\section{Key Words}

Porcine oocyte Polyadenylation - Maturation - Maternal gene $\cdot$ Chromosome alignment

\begin{abstract}
Aims: This study aims to clarify the effects of polyadenylation status on M-phase promoting factors (MPFs) during in vitro porcine oocyte maturation. Methods: In this study, porcine follicular oocytes from large follicles ( $>5$ millimeter $(\mathrm{mm})$ ) and small follicles $(<3 \mathrm{~mm}$ ) were examined at different follicular developmental stages. The polyadenylation of maternal mRNAs was inhibited by the addition of 3'-deoxyadenosine (3'-da) during the germinal vesicle (GV)(0 h), GV breakdown (GVBD)(18 h), metaphase I (MI)(28 h), and metaphase II (MII) (44 h) stages. In addition, the expression levels and poly-(A) tail lengths of the maternal mRNAs Cyclin B1 and cell division cycle $2(C d c 2)$ were determined by real-time quantitative PCR. Immunofluorescence was used to assess spindle formation and chromosome alignment in the examined oocytes. Results: In large-follicle oocytes, the effects of inhibiting polyadenylation caused the percentage of mature to be significantly lower for the treated group than for the untreated group $(p<0.01)$. 3'-da can significantly improve the rate of small oocyte maturation in vitro and inhibits $C d c 2$ polyadenylation. Cyclin B1 plays a significant role in promoting the maturation of large-follicle oocytes. Polyadenylation contributes to the formation of dominant follicles and facilitates the selection of dominant follicles. However, the inhibition of adenylation affected spindle formation-related propulsion and chromosome alignment in both large- and small-follicle oocytes. The first polar body could not be extruded in certain large follicles. Conclusions: 3 '-da can significantly improve the rate of small oocyte maturation in vitro, but it can also affect spindle formation-related propulsion and chromosome alignment.
\end{abstract}

H. Liu and Y. Gao contributed equally to this article. 


\section{Introduction}

Animal ovaries contain numerous follicular oocytes in different developmental stages, and a follicular cavity is absent for the majority of these oocytes. At present, most of the oocytes that are used for research applications are acquired from antral follicles; however, extremely few follicles in mammalian ovaries fully mature to participate in ovulation. Approximately $99.9 \%$ of follicles undergo atresia and degradation during the preantral follicle stage. A large number of preantral follicles and oocytes within primordial follicles are not appropriately utilized, which is a phenomenon that undoubtedly represents a great loss of genetic and breeding resources for animals. The messenger RNA (mRNA) and ribosomal RNA (rRNA) molecules that are responsible for the completion of synthesis and metabolism during the oocyte cell cycle are stored in the oocyte cytoplasm, and these mRNA and rRNA molecules are largely synthesized during the course of oocyte growth. Therefore, the extent of oocyte growth, which may be represented by follicular size, is closely related to in vitro oocyte maturation. Many studies have demonstrated that as follicular volume increases, the oocyte maturation rate, fertilization rate, and blastocyst rate will increase [1-3].

In mammalian oocytes, gene expression is mainly regulated at the post- transcriptional level; in particular, this regulation primarily occurs through cytoplasmic polyadenylation, a process that plays an important role in protecting mRNAs from degradation and stimulating the translation of these mRNAs [4]. During the meiotic maturation of mammalian oocytes, the mother must accumulate more energy than is required for in vitro oocyte maturation and early embryonic development. Further studies are required to clarify the molecular mechanisms of in vitro mammalian oocyte maturation, which depend on a precise sequence of changes in maternal gene expression. However, oocytes contain a limited number of mRNAs; as a result, it is difficult to conduct genetic analysis studies in oocytes. During the early stages of zygotic genome activation, virtually no transcription occurs, and gene expression is regulated at the post-transcriptional level, particularly via cytoplasmic polyadenylation. This process plays an extremely important role in preventing mRNA degradation and stimulating translation. The translation potential of maternal mRNAs primarily depends on the poly-(A) tail length, and deadenylation is typically the first sign of mRNA degradation (reviewed by Bettegowda and Smith, 2007). It can be inferred that the functions of polyadenylation/deadenylation may not only affect mRNA stability and translation efficiency during the course of oocyte maturation, but they may also affect mRNA degradation during early embryonic development, either before or after genome activation. Krischek and Meinecke demonstrated that the maturation of bovine oocytes requires the polyadenylation of maternal mRNAs [5]. The overexpression of poly-(A) polymerase (PAP) in vivo intensifies cellular polyadenylation levels, thereby increasing the death rates of Drosophila embryos [6].

To date, studies have only characterized a small number of maternal gene products in human or mouse oocytes. Among the transcription products of these maternal genes, Cyclin $B 1$ is regarded as a marker of female germ cells. $C$-mos is an oncogene that is regarded as an oocyte maturation regulator in humans [7], mice [8], and pigs [9]. Real-time polymerase chain reaction (PCR) and poly-(A) tests (PATs) have been used for the examination of Cyclin $B 1$, and one study has investigated the differential expression of C-mos, cyclin B, GDF9 and BMP15 four maternal genes during porcine oocyte maturation [10].

To date, no researchers have examined how polyadenylation inhibition affects the maturation rate and maternal gene expression in follicles of various sizes. In this study, these two topics were examined to better understand the molecular mechanisms of oocyte maturation and the effects of polyadenylation inhibition on the spindle that is formed by oocyte chromosomes. 


\section{Cellular Physiology Cell Physiol Biochem 2016;39:1735-1745

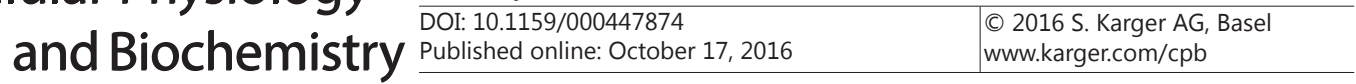

Liu et al.: The Effects of Polyadenylation Status on MPFs

\section{Materials and Methods}

Oocyte maturation

Ovaries were collected from freshly slaughtered sows, placed in normal saline containing $75 \mathrm{mg} /$ $\mathrm{ml}$ of penicillin $\mathrm{G}$ and $50 \mathrm{mg} / \mathrm{ml}$ streptomycin sulfate at $37^{\circ} \mathrm{C}$, and transported to the laboratory within two hours. In a sterile operating room, cumulus-oocyte complexes (COCs) were extracted using a syringe with an 18-gauge needle. Microscopic observation was used to select COCs with uniform cytoplasms and intact zonae pellucidae that were wrapped in three to five layers of granulosa cells. The selected COCs were washed twice with HEPES-buffered Tyrode's medium containing 0.1\% (w:v) polyvinyl alcohol (HEPES-TLPVA) and twice with the culture medium. Tissue culture medium 199 (TCM-199) was used, to which $0.1 \%$ PVA (w/v), $3.05 \mathrm{mM}$ D-glucose, $0.91 \mathrm{mM}$ sodium pyruvate, $0.57 \mathrm{mM}$ cysteine, $10 \mathrm{ng} / \mathrm{ml}$ epidermal growth factor (EGF; Sigma, St. Louis, MO, USA), $10 \mathrm{IU} / \mathrm{ml}$ PMSG, $10 \mathrm{IU} / \mathrm{ml} \mathrm{hCG,} 75 \mathrm{mg} / \mathrm{ml}$ penicillin G, and $50 \mathrm{mg} / \mathrm{ml}$ streptomycin sulfate had been added. The culture conditions included an atmosphere containing $5 \% \mathrm{CO}_{2}$, maximum relative humidity, and a temperature of $39^{\circ} \mathrm{C}$.

\section{Experimental grouping and treatment concentrations}

Large and small follicles were divided into experimental (treated) and control (untreated) groups. Large follicles were defined as follicles with diameters of greater than five millimeters, and small follicles were defined as follicles with diameters of less than three millimeters. The concentration of 3 '-deoxyadenosine ( 3 '-da) that was added to the treated group was $2 \mu \mathrm{g} / \mathrm{ml}$. COCs were collected at 0 hours (h), $18 \mathrm{~h}, 28 \mathrm{~h}$, and $44 \mathrm{~h}$ and were treated for 2 minutes ( $\mathrm{min}$ ) with $0.1 \%$ hyaluronidase. Naked oocytes were collected and stored in liquid nitrogen for further gene expression analyses. Each group consisted of 40 oocytes.

\section{Fluorescence quantitative PCR and PAT}

After RNA was extracted from the porcine oocytes during the four developmental stages, which included the germinal vesicle (GV), GV breakdown (GVBD), metaphase I (MI), and metaphase II (MII) stages, reverse transcription was conducted using a reverse transcription kit from Toyobo. Primers for fluorescent quantitative PCR were designed using Primer 5.0 software. Green fluorescent dye from Toyobo was used for PCR amplifications. Fluorescent quantitative samples were amplified in triplicate to ensure an appropriate repetition of the technique. For each group of samples, internal controls from the same time period were established using $\beta$-actin as an internal reference. Fluorescence quantitative PCR data were analyzed using the $2^{-\Delta \Delta C T}$ method. The following approach was employed to determine the poly-(A) tail lengths from the PCR results: the first-strand cDNA was synthesized using reverse transcription of isolated mRNAs using Oligo(dT)-Anchor as the primer (5'-GCGAGCTCCGCGGCC-GCGT12-3' Salles and Strickland, 1999). A subsequent PCR was performed using Oligo(dT)-Anchor and gene-specific upstream primers for the maternal test transcripts. The experiments were repeated three times.

\section{Western-blot}

Using protein extraction kit from Toyobo extracted total protein from the porcine oocytes during the four developmental stages, which included the germinal vesicle (GV), GV breakdown (GVBD), metaphase I (MI), and metaphase II (MII) stages, and then detected the expression of Cyclin B1 and Cdc2 in porcine oocytes of each group by Western-blot.

\section{Immunofluorescence}

The oocytes were washed with phosphate-buffered saline (PBS) with shaking. Rabbit anti- $\alpha$-Tubulin antibody (1:200) was added dropwise, and the oocytes were incubated at $37^{\circ} \mathrm{C}$ in a humidified chamber for $120 \mathrm{~min}$. The oocytes were then removed from the chamber and washed three times with PBS with shaking. A fluorescein isothiocyanate (FITC)-labeled goat anti-rabbit secondary antibody (1:500) was added dropwise, and the samples were incubated at $37^{\circ} \mathrm{C}$ for $120 \mathrm{~min}$. Following this incubation, the oocytes were again washed three times with PBS with shaking. The samples were then stained by adding propidium iodide (PI) (1:100), and the oocytes were incubated at $37^{\circ} \mathrm{C}$ for $10 \mathrm{~min}$. The oocytes were then washed with PBS with shaking. After mounting, the samples were observed using a fluorescence microscope. 


\section{Results}

\section{Effects of polyadenylation on oocyte maturation rates}

The maturation rates for 3'-da-treated and untreated large-follicle oocytes were $65.97 \%$ and $88.9 \%$, respectively; this difference in maturation rates was determined to be significant $(p<0.01)$. The treated and untreated large-follicle oocytes did not exhibit significant differences in the percentage of dead or immature oocytes $(p>0.05)$. The percentage of mature for 3 '-da-treated and untreated small-follicle oocytes were $61.7 \%$ and $48.4 \%$, respectively; this difference in the percentage of mature was significant $(p<0.05)$. The treated and untreated small-follicle oocytes did not exhibit significant differences in the percentage of dead or immature ( $p>0.05)$ (Fig. 1A and 1B). Large- and small-follicle oocytes in the control group exhibited significantly different the percentage of mature $(p<0.05)$. However, the percentage of mature of 3 '-da-treated large- and small-follicle oocytes did not significantly differ $(p>0.05)$ (Fig. 1C and 1D).

\section{Effects of polyadenylation on maternal gene expression in oocytes}

Treatment with the polyadenylation inhibitor 3'-da produced decreases in Cyclin B1 expression in large-follicle porcine oocytes during the MI and MII stages and in small-follicle porcine oocytes during the GVBD, MI, and MII stages relative to the corresponding control oocytes (Fig. 2A). In large-follicle porcine oocytes, Cdc2 expression was lower in the 3 '-da group than in the control group during the GVBD, MI, and MII stages; however, in smallfollicle porcine oocytes, $C d c 2$ expression was higher in the 3 '-da group than in the control group during the GVBD, MI, and MII stages (Fig. 2B).

Fig. 1. (A) A comparison of the in $v i-$ tro the percentage of mature for large-follicle oocytes that were treated with 3'-da and untreated large-follicle oocytes. The three categories indicate the percentage of immature, mature, and dead in the oocytes. (B) A comparison of the in vitro the percentage of mature for small-follicle oocytes that were treated with 3'-da and untreated small-follicle oocytes. The three
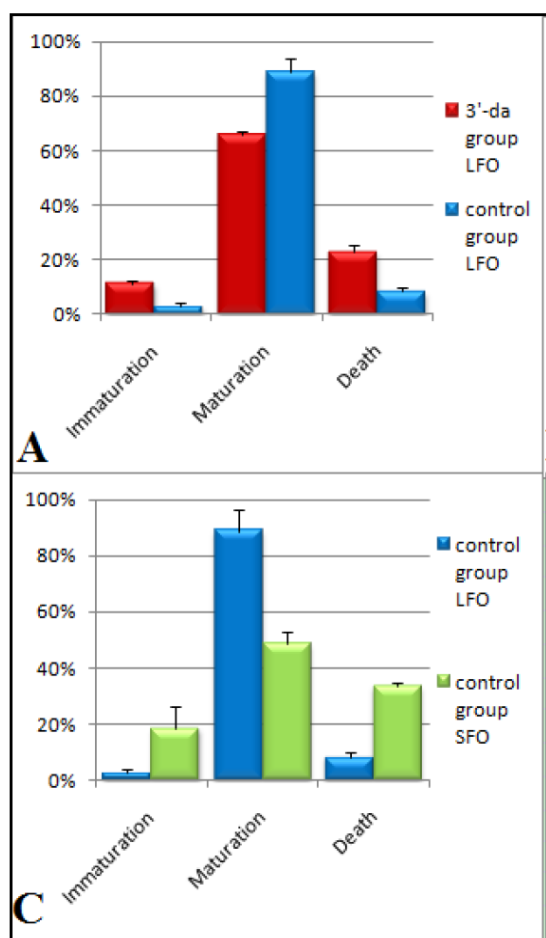

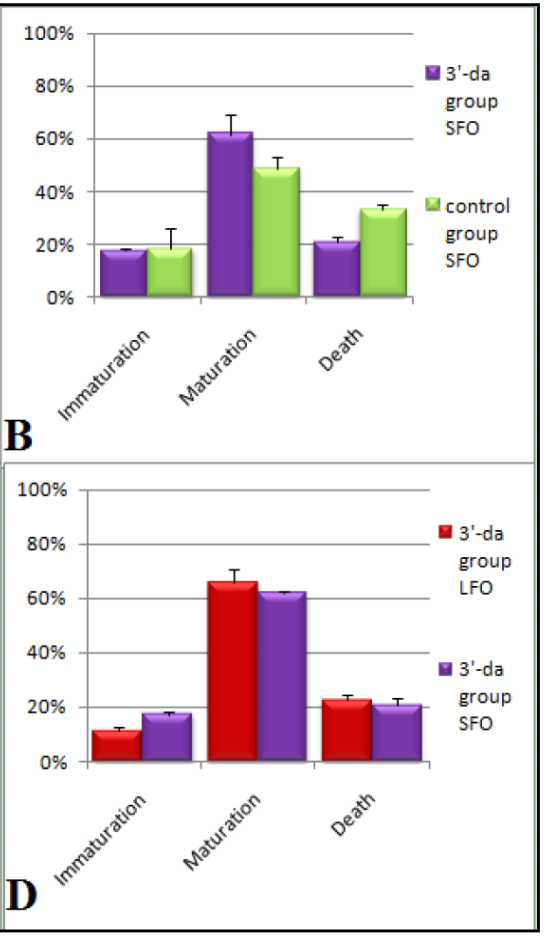
categories indicate the percentage of immature, mature, and dead in the oocytes. (C) A comparison of the in vitro the percentage of mature for untreated large-follicle oocytes and untreated small-follicle oocytes. The three categories indicate the percentage of immature, mature, and dead in the oocytes. (D) A comparison of the in vitro the percentage of mature for 3'-da-treated large-follicle oocytes and 3'-da-treated small-follicle oocytes. The three categories indicate the percentage of immature, mature, and dead in the oocytes. 
Fig. 2. Real-time fluorescence quantitative PCR results indicating maternal gene expression levels in experimental groups of oocytes that were treated with the polyadenylation inhibitor 3'-da and untreated oocyte control groups. (A) $\mathrm{Cy}$ clin $B 1$ expression in the experimental and control groups for large- and small-follicle oocytes during the GV, GVBD, MI, and MII stages. (B) Cdc2 expression in the experimental and control groups for large- and small-follicle oocytes during the GV, GVBD, MI, and MII

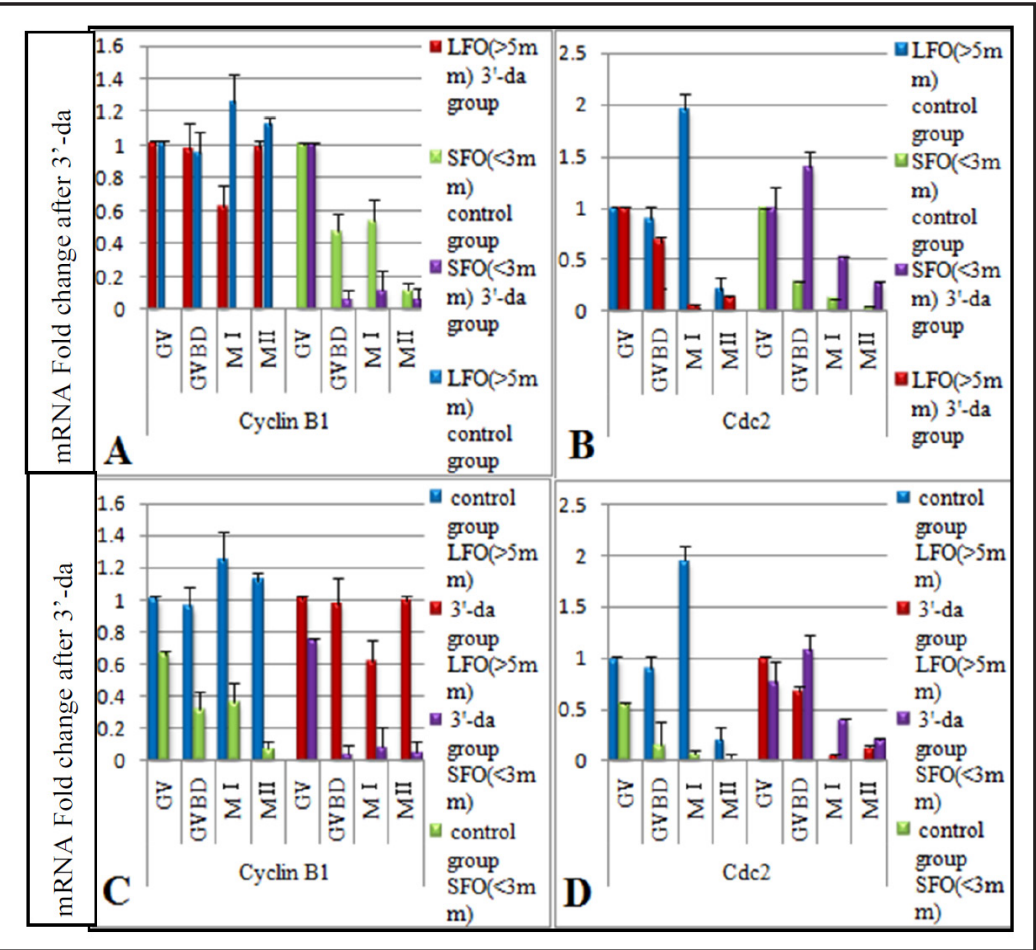
stages. (C) Cyclin B1 expression in large- and small-follicle oocytes for the experimental and control groups during the GV, GVBD, MI, and MII stages. (D) Cdc2 expression in large- and small-follicle oocytes for the experimental and control groups during the GV, GVBD, MI, and MII stages.

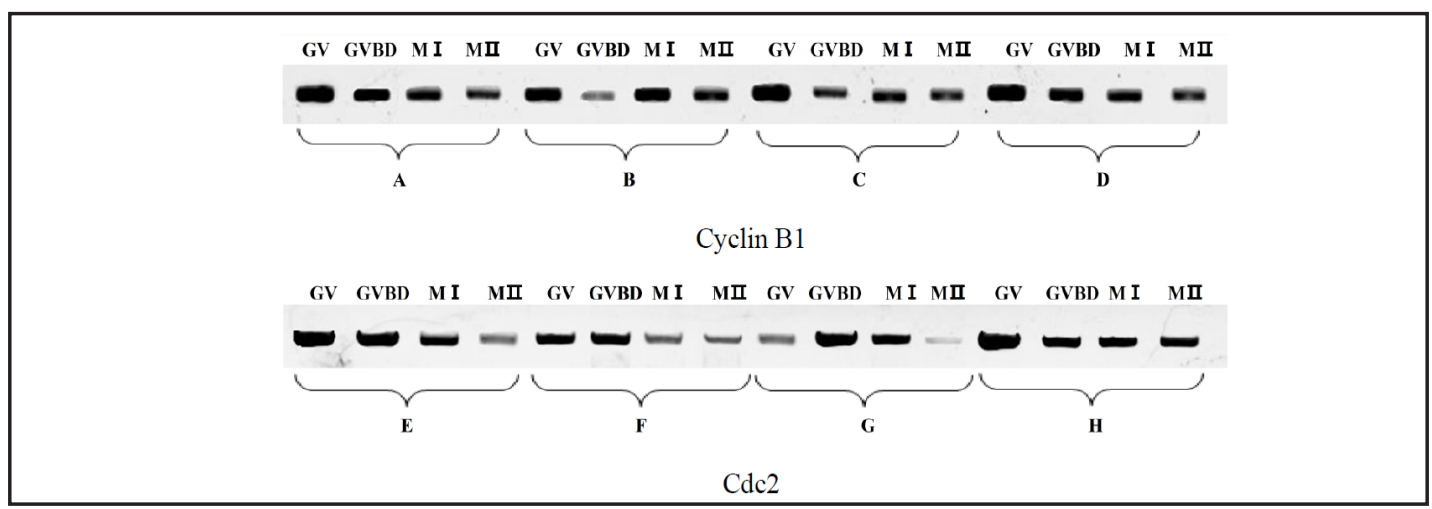

Fig. 3. Western blot results indicating maternal gene expression levels in experimental groups of oocytes that were treated with the polyadenylation inhibitor 3 '-da and untreated oocyte control groups. (A) Cyclin B1 expression in the experimental groups for large-follicle oocytes during the GV, GVBD, MI, and MII stages. (B) Cyclin B1 expression in the control groups for large-follicle oocytes during the GV, GVBD, MI, and MII stages. (C) Cyclin B1 expression in the experimental groups for small-follicle oocytes during the GV, GVBD, MI, and MII stages. (D) Cyclin B1 expression in the control groups for small-follicle oocytes during the GV, GVBD, MI, and MII stages. (E) Cdc2 expression in the experimental groups for largefollicle oocytes during the GV, GVBD, MI, and MII stages. (F) Cdc2 expression in the control groups for large-follicle oocytes during the GV, GVBD, MI, and MII stages. (G) Cdc2 expression in the experimental groups for small-follicle oocytes during the GV, GVBD, MI, and MII stages. $(\mathrm{H}) \mathrm{Cdc2}$ expression in the control groups for small-follicle oocytes during the GV, GVBD, MI, and MII stages. 


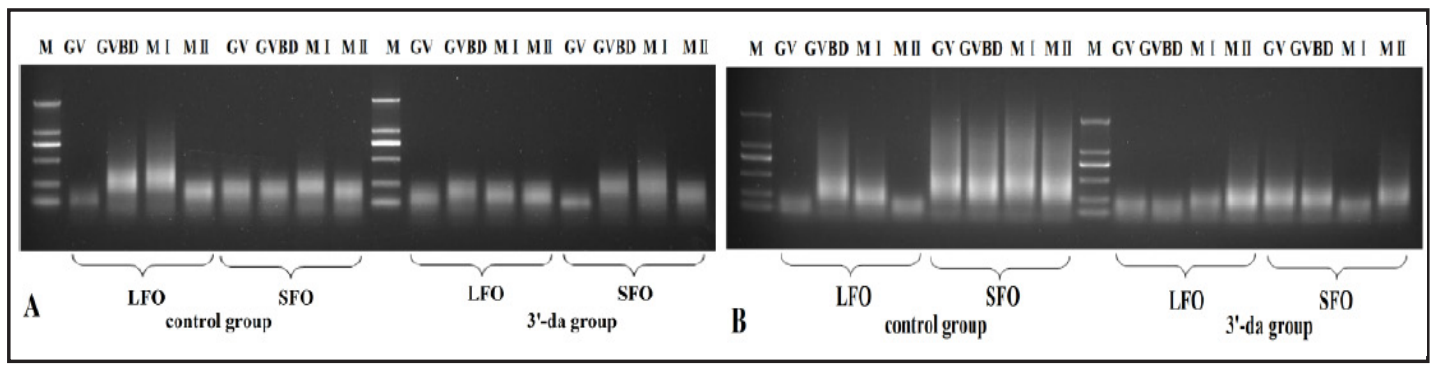

Fig. 4. PAT results indicating the poly-(A) tail lengths of the Cyclin B1 and Cdc2 mRNAs during the GVBD, MI, and MII stages. (A) Cyclin B1 mRNA for large- and small-follicle oocytes in untreated control group oocytes and experimental group oocytes that were treated with the polyadenylation inhibitor 3'-da. (B) Cdc2 mRNA for large- and small-follicle oocytes in untreated control-group oocytes and experimental group oocytes that have been treated with the polyadenylation inhibitor 3 '-da.

Fig. 5. Immunofluorescence results indicating the progression of spindle formation and chromosome alignment during the GV, GVBD, MI, and MII stages. (A) Large-follicle oocytes from the control group during the GV, GVBD, MI, and MII stages. (B) Large-follicle oocytes in the experimental group that had been treated with the polyadenylation inhibitor 3 '-da during the GV, GVBD, MI, and MII stages. (C) Small-follicle oocytes from the control group during the GV, GVBD, MI, and MII stages. (D) Small-follicle oocytes in the experimental group that had been treated with the po-

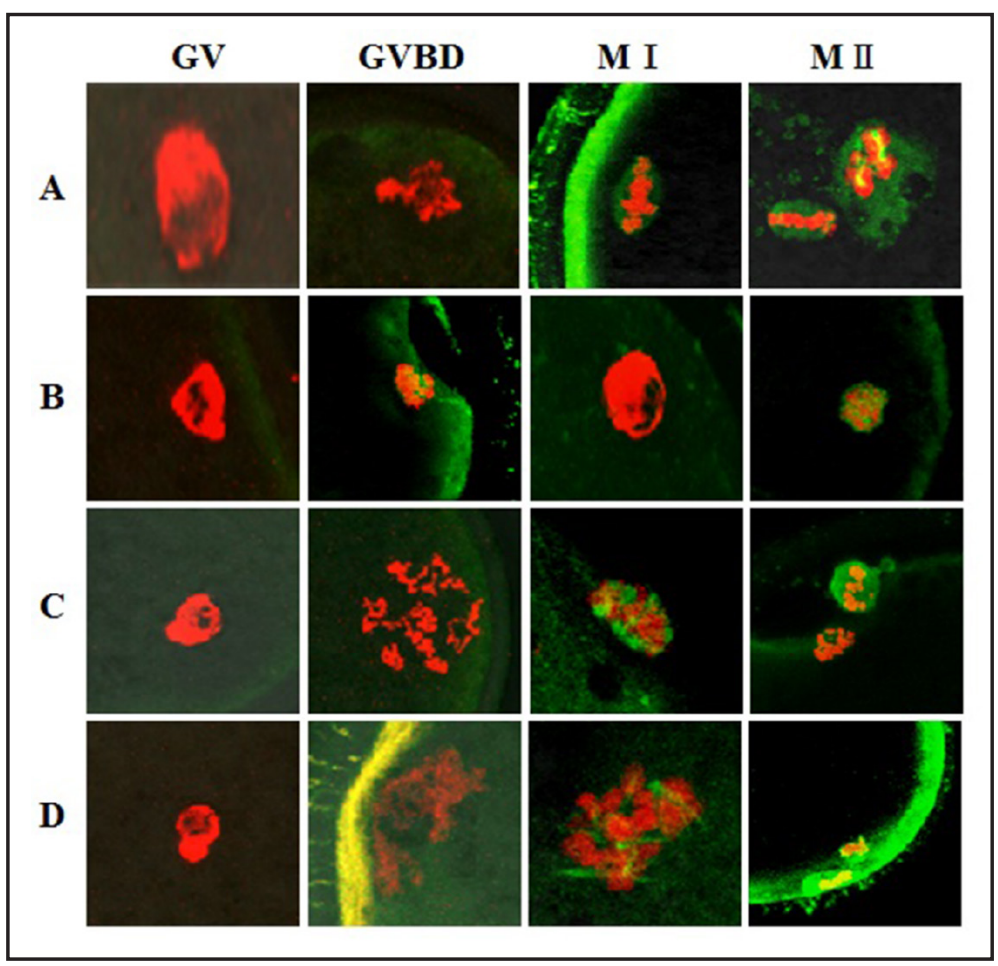
lyadenylation inhibitor 3'-da during the GV, GVBD, MI, and MII stages. ( $\alpha$-Tubulin in green; chromosomes in red).

Differences between large- and small-follicle oocytes with respect to maternal gene expression

In the control group oocytes, Cyclin B1 expression in small-follicle oocytes was lower than in large-follicle oocytes during the GV, GVBD, MI, and MII stages. In oocytes treated with the polyadenylation inhibitor 3'-da, Cyclin B1 expression was much lower in the smallfollicle oocytes relative to the large-follicle oocytes during the GVBD, MI, and MII stages. In the control group oocytes, $C d c 2$ expression was lower in small-follicle oocytes than in large-follicle oocytes during the GV, GVBD, MI and MII stages. In oocytes treated with the polyadenylation inhibitor 3 '-da, $C d c 2$ expression was lower in the small-follicle oocytes than in the large-follicle oocytes during the GV stages but was higher in the small-follicle oocytes

\section{KARGER}


than in the large-follicle oocytes during the GVBD, MI and MII stages.The results of Westernblot show that the expression of $C y c l i n B 1$ and $C d c 2$ in the experimental and the control group were basically consistent with the results of fluorescence quantitative PCR test in the each period group porcine oocytes(Fig. 3).

\section{Effects of polyadenylation on the poly-(A) tails of maternal $m R N A s$}

The PAT method was used to determine the lengths of the poly-(A) tails on transcripts of the maternal genes Cyclin B1 and $C d c 2$ in 3'-da-treated porcine oocytes and normal (untreated) porcine oocytes during the GVBD, MI, and MII stages. We detect smaller bands in experimental oocytes that were treated by the polyadenylation inhibitor 3 '-da with the control oocytes with respect to the poly-(A) tail lengths of Cyclin B1. Relative to untreated large-follicle porcine oocytes, large-follicle porcine oocytes that were treated with the polyadenylation inhibitor 3'-da produced smaller $C d c 2$ bands during the GVBD and MI stages. Relative to untreated small-follicle porcine oocytes, small-follicle porcine oocytes that were treated with the polyadenylation inhibitor 3'-da produced smaller $C d c 2$ bands during the GV, GVBD, MI, and MII stages (Fig. 4).

Effects of polyadenylation on spindle formation and chromosome alignment at various developmental stages in the oocyte

Immunofluorescence was used to detect $\alpha$-Tubulin protein, which was stained with PI. The progressions of spindle formation and chromosome alignment were relatively normal in untreated large- and small-follicle oocytes during the GV, GVBD, MI, and MII stages. In smallfollicle oocytes, treatment with the polyadenylation inhibitor 3 '-da significantly disrupted spindle formation and affected chromosome alignment (Fig. 5D, MI). In these treated smallfollicle oocytes, during the MII stage, the first polar body could be extruded appropriately; however, the spindle was greatly affected by the 3 '-da treatment (Fig. 5D, MII). In the large-follicle oocytes, the addition of the polyadenylation inhibitor 3 '-da disrupted spindle formation somewhat, although this disruption was not particularly obvious (Fig. 5B, MI). As a result of this disruption, the first polar body could not be extruded in certain large follicles (Fig. 5B, MII).

\section{Discussion}

The addition of 3'-da effectively inhibited mRNA polyadenylation in large-follicle oocytes, indicating that polyadenylation plays a role in the maturation process of these oocytes. mRNA polyadenylation levels are greater in large-follicle oocytes than in smallfollicle oocytes, indicating that polyadenylation promotes the formation of dominant follicles and is conducive to the selection of dominant follicles. The PAT results also suggest that polyadenylation plays a role in promoting oocyte maturation.

The polyadenylation status of the maternal gene transcripts Cyclin B1 and Cdc2 determines the expression levels of these genes in oocytes. These genes are involved in the process of selecting dominant follicles from large and small follicles as well as play key roles in promoting oocyte maturation. In particular, Cyclin B1 plays a larger role in the maturation of large-follicle oocytes than in the maturation of small-follicle oocytes.

The inhibition of maternal mRNA polyadenylation produced varying effects on the progression of spindle formation and chromosome alignment in large- and small-follicle oocytes. In particular, although these effects appeared to be less extensive in large-follicle oocytes relative to small-follicle oocytes, the first polar body could not be extruded by certain large follicular oocytes as a result of spindle formation and chromosome alignment problems, with a corresponding decrease in the rate of large-follicle oocyte maturation. In small-follicle oocytes, although the maturation rate increased after the inhibition of maternal mRNA polyadenylation, this inhibition greatly disrupted spindle formation and somewhat affected chromosome alignment. Thus, despite the increase in the rate of small- 
follicle oocyte maturation that resulted from inhibiting maternal mRNA polyadenylation, the abnormalities in the progression of spindle formation and chromosome alignment that are caused by this inhibition are likely to affect subsequent experimental studies of 3'-datreated small-follicle oocytes.

Maturation promoting factor (MPF) plays an extremely important role in oocyte maturation. Through the regulation of histone phosphorylation, MPF enables chromatin condensation and alignment. MPF activity is critical to the precise regulation of mitosis. MPF is a heterodimeric protein that is composed of a smaller subunit, $C d c 2$, and a larger subunit, cyclin B1. cyclin B1 can regulate MPF activity; thus, changes in cyclin B1 structure reduce MPF activity, thereby promoting cell death.

Cyclin B1 polyadenylation may be related to the first peak of MPF activity that occurs during the cell cycle, which occurs around MI (10-12 h after the onset of the maturation period). It has also been shown that, in the culture conditions that were used in this study, the inhibition of polyadenylation prevents meiosis progression, especially leading up to the MI stage, while the inhibition of transcription does not [11]. A previous study shows a link between the cytoplasmic polyadenylation of cyclin B1 and the translation and appearance of cyclin B1 protein before in vitro maturation [12]. In cooperation with the CPEB-maskin complex, the master regulator that is common to CPE-containing mRNAs is XPum. XPum acts as a specific regulator that determines the timing of the translational activation of cyclin $B 1$ mRNA by its release from phosphorylated CPEB during oocyte maturation [13]. The phosphorylation of CPEB mediates its dissociation from ribonucleoprotein complexes prior to its degradation [14].

When Aurora Kinase A activity is inhibited, neither cyclin B1 mRNA polyadenylation nor its translation are impaired, and this kinase is unlikely to be involved in CPEB1activating phosphorylation [15]. Insulin-like growth factor 2 mRNA-binding protein 3 may be involved in zebrafish oocyte maturation as a novel cyclin B1 mRNA-binding protein that represses translation in immature oocytes [16]. cyclin $B 1$ is posttranscriptionally regulated by cytoplasmic polyadenylation during porcine oocyte maturation. Further, the decreased expression of maternal cyclin B1 and Cdc2 at the mRNA or protein level in developmentally incompetent oocytes and embryos is responsible for, at least in part, a profound defect in further embryonic development [17].

The expression level of $C d c 2$ was increased in small-follicle oocytes that were treated with 3'-dA, suggesting that polyadenylation inhibition in small-follicle oocytes promotes MPF activity. We speculate that $C d c 2$ may promote small-follicle oocyte maturation, but the spindle fails to reach the mature oocyte form that is induced by treatment with 3 'dA. Zhang et al. shows that cyclin B1 was posttranscriptionally regulated by cytoplasmic polyadenylation during porcine oocyte maturation [10].

The levels of cyclin B1 and Cdc2 were decreased in large-follicle oocytes that were treated with 3 '- $\mathrm{dA}$, suggesting that the process of large-follicle oocyte maturation becomes slow as a result of 3'-dA treatment, while the spindle in large-follicle oocytes was not affected by treatment.

In this study, the length of the cyclin B1 poly(A) tail in the 3'-dA-treated group was altered during normal IVM, which is consistent with the result of a previous study [17]. JUAN $\mathrm{M}$ et al. used 3'-dA-treated bovine oocytes to detect changes in cyclin B1 poly(A) tails; cyclin $B 1$ in immature oocytes appeared as a short smear with RACE-PAT that lengthened after 24 $\mathrm{h}$ of incubation in the maturation medium [18].

While rapamycin treatment affected global protein synthesis, the drug altered neither the specific translation of cyclin B mRNA nor the expression of the Mos protein. The expression of these two proteins was correlated with the phosphorylation and the dissociation of the cytoplasmic polyadenylation element-binding protein from eIF4E [19]. A polyadenylation inhibitor blocked pig oocyte maturation in vitro by one or more of the following actions: (1) inactivation of MAPK and MPF in oocytes, especially at the late stages (MI and MII); (2) prevention of cumulus cell expansion via the inactivation of cellular MAPK; and (3) inhibition of the maternal mRNA polyadenylation process, which in reverse, disrupted maternal 
mRNA patterns in the in vitro pig oocyte maturation process [20]. Although the factors that regulate the translation of maternal mRNAs are highly conserved, the target sequences and overall sequence architecture within the 3'UTR of cyclin B1 mRNA have diverged to affect translational efficiency, perhaps to optimize levels of cyclin $B 1$ protein that are required by these different species during their earliest embryonic cell divisions [21].

R0-3306 efficiently blocks and synchronizes the meiotic progression of porcine oocytes at the GV stage without affecting their meiotic and cytoplasmic maturation [22]. The observed increases in polyadenylated transcript abundance within the RNA-Seq data are likely due to $\mathrm{CP}$, providing novel insight into targeted transcripts and the resultant differential gene expression profiles that contribute to oocyte maturation [23]. In another study, both $C d c 2$ and GDF9 mRNA and polyadenylation levels showed significantly higher expression in the drug treatment groups [20].

The inhibition of maternal mRNA polyadenylation caused significant increases in $C d c 2$ gene expression in small-follicle oocytes, suggesting that $C d c 2$ plays a role in promoting the extrusion of the first polar body.

Cyclin B1 mRNA should theoretically be polyadenylated soon after the beginning of oocyte maturation. We speculate that some related gene polyadenylations inhibited the synthesis of or degraded cyclin B1 mRNA after the addition of 3 'da. Moreover, because the oocytes were used in the experiments over a fixed period, it is likely that the cell cycle progression was impeded or delayed. Therefore, we detected small changes in cyclin B1 mRNA polyadenylation in small oocytes after 3'-da treatment with control oocytes. Komrskova's research demonstrated no obvious changes in cyclin $B 1$ mRNA polyadenylation with poly(A) following the addition of MLN8237[15].

We observed that the polyadenylations of some maturation-related genes were inhibited in the small oocytes following 3'-da treatment, which promoted small oocyte maturation. These findings indicate that the polyadenylations of some maturation genes prevented or slowed the small oocyte maturation process.

Polar body extrusion in the small oocytes was promoted after the 3'-da treatment, but this treatment did not efficiently promote spindle formation. We determined that polyadenylation inhibited the effects on polar body extrusion-related genes and thus inhibited polar body extrusion in the small oocytes. Some genes, such as cyclin B1, play important roles in spindle formation during oocyte maturation. However, the oocyte cycle was halted or diminished in the small oocytes after 3'-da treatment, which caused the disordering or slowing of spindle formation in the small oocytes. However, gene regulation was maintained during the maturation of the large oocytes. Therefore, although the polyadenylation of some genes was inhibited following 3'-da treatment, normal spindle formation was still achieved.

Pigs not only provide food for humans but also serve as a model animal for studies on biological and reproductive medicine. At present, basic techniques for the in vitro maturation of porcine oocytes have been utilized in studies of oocyte reproductive biology (e.g., in vitro fertilization and test-tube embryos) and extended to practical applications (e.g., the production of cloned embryos and animals) [10]. Because human oocytes are difficult to obtain and variety of factors was associated with number of human embryos produced such as AMH [24], as there is a close genetic relationship between pigs and humans, this study examined porcine oocytes, where the investigation of maturation rates, chromosome alignment, and spindle formation should allow for similar conclusions to be drawn for human oocytes. Thus, the current study helps to elucidate the molecular mechanisms of human oocyte maturation, enabling individuals to better understand and utilize factors that influence human oocyte maturation.

\section{Abbreviations}

Cdc2 (cell division cycle 2); mRNA (messenger RNA); rRNA (ribosomal RNA ); PAP (poly(A) polymerase); PCR (polymerase chain reaction); PAT (poly-(A) test); COCs (cumulus- 
oocyte complexes); Hepes-TL-PVA (Hepes-buffered Tyrode's medium containing $0.1 \%$ polyvinyl alcohol); TCM-199 (tissue culture medium 199); EGF (epidermal growth factor); GV (germinal vesicle); GVBD (GV breakdown); MI (metaphase I); MII (metaphase II); PBS (phosphate-buffered saline); FITC (fluorescein isothiocyanate); PI (propidium iodide); MPF (Maturation promoting factor).

\section{Acknowledgments}

This work was supported by the earmarked fund for Modern Agro-industry Technology Research Systerm (CARS-38), Youth Science Research Fund Program from Jilin Province (20140520176JH), the 55 ${ }^{\text {th }}$ Batch Face Funding Program from China Post-doctoral Science Fund (2014M551197), and National Natural Science Foundation (31201761).

\section{References}

1 Yoon JD, Jeon Y, Cai L, Hwang SU, Kim E, Lee E, Kim DY, Hyun SH: Effects of coculture with cumulus-derived somatic cells on in vitro maturation of porcine oocytes. Theriogenology 2015; 83:294-305.

2 Dias FC, Dadarwal D, Adams GP, Mrigank H, Mapletoft RJ, Singh J: Length of the follicular growing phase and oocyte competence in beef heifers. Theriogenology 2013;79:1177-1183.

3 Kim J, You J, Hyun SH, Lee G, Lim J, Lee E: Developmental competence of morphologically poor oocytes in relation to follicular size and oocyte diameter in the pig. Mol Reprod Dev 2010;77:330-339.

4 Bettegowda A, Smith GW: Mechanisms of maternal mRNA regulation: implications for mammalian early embryonic development. Front Biosci 2007;12:3713-3726.

$5 \quad$ Krischek C, Meinecke B: In vitro maturation of bovine oocytes requires polyadenylation of mRNAs coding proteins for chromatin condensation, spindle assembly, MPF and MAP kinase activation. Anim Reprod Sci 2002;73:129-140.

6 Juge F, Zaessinger S, Temme C, Wahle E, Simonelig M: Control of poly(A) polymerase level is essential to cytoplasmic polyadenylation and early development in Drosophila. EMBO J 2002;21: 6603-6613.

7 Hashiba Y, Asada Y, Heikinheimo O, Lanzendorf SE, Mizutani S: Microinjection of antisense c-mos oligonucleotides prevents the progression of meiosis in human and hamster oocytes. Fertil Steril 2001;76:143-147.

8 O'Keefe SJ, Wolfes H, Kiessling AA, Cooper GM: Microinjection of antisense c-mos oligonucleotides prevents meiosis II in the maturing mouse egg. Proc Natl Acad Sci U S A 1989;86:7038-7042.

9 Ohashi S, Naito K, Sugiura K, Iwamori N, Goto S, Naruoka H, Tojo H: Analyses of mitogen-activated protein kinase function in the maturation of porcine oocytes. Biol Reprod 2003;68:604-609.

10 Zhang DX, Cui XS, Kim NH: Involvement of polyadenylation status on maternal gene expression during in vitro maturation of porcine oocytes. Mol Reprod Dev 2009;76:881-889.

11 Traverso JM, Donnay I, Lequarre AS: Effects of polyadenylation inhibition on meiosis progression in relation to the polyadenylation status of cyclins A2 and B1 during in vitro maturation of bovine oocytes. Mol Reprod Dev 2005;71:107-114.

12 Tremblay K, Vigneault C, McGraw S, Sirard MA: Expression of cyclin B1 messenger RNA isoforms and initiation of cytoplasmic polyadenylation in the bovine oocyte. Biol Reprod 2005;72:1037-1044.

13 Nakahata S, Kotani T, Mita K, Kawasaki T, Katsu Y, Nagahama Y, Yamashita M: Involvement of Xenopus Pumilio in the translational regulation that is specific to cyclin B1 mRNA during oocyte maturation. Mech Dev 2003;120:865-880.

14 Thom G, Minshall N, Git A, Argasinska J, Standart N: Role of cdc2 kinase phosphorylation and conserved $\mathrm{N}$-terminal proteolysis motifs in cytoplasmic polyadenylation-element-binding protein (CPEB) complex dissociation and degradation. Biochem J 2003;370:91-100.

15 Komrskova P, Susor A, Malik R, Prochazkova B, Liskova L, Supolikova J, Hladky S, Kubelka M: Aurora kinase A is not involved in CPEB1 phosphorylation and cyclin B1 mRNA polyadenylation during meiotic maturation of porcine oocytes. PLoS One 2014;9:e101222. 


\section{Cellular Physiology Cell Physiol Biochem 2016;39:1735-1745

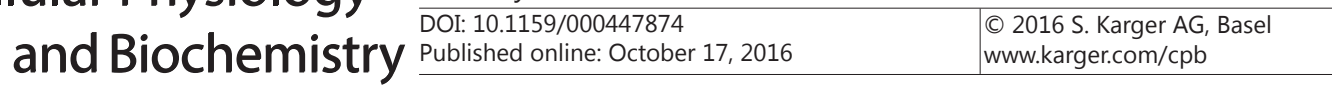 \\ Liu et al.: The Effects of Polyadenylation Status on MPFs}

16 Takahashi K, Kotani T, Katsu Y, Yamashita M: Possible involvement of insulin-like growth factor 2 mRNAbinding protein 3 in zebrafish oocyte maturation as a novel cyclin B1 mRNA-binding protein that represses the translation in immature oocytes. Biochem Biophys Res Commun 2014; 448:22-27.

17 Zhang DX, Cui XS, Kim NH: Molecular characterization and polyadenylation- regulated expression of cyclin B1 and Cdc2 in porcine oocytes and early parthenotes. Mol Reprod Dev 2010;77:38-50.

18 Traverso JM, Donnay I, Lequarre A-S: Effects of Polyadenylation Inhibition on Meiosis Progression in Relation to the Polyadenylation Status of Cyclins A2 and B1 During In Vitro Maturation of Bovine Oocytes. Mol Reprod Develop 2005;71:107-114.

19 Lapasset L, Pradet-Balade B, Vergé V, Lozano JC, Oulhen N, Cormier P, Peaucellier G: Cyclin B synthesis and rapamycin-sensitive regulation of protein synthesis during starfish oocyte meiotic divisions. Mol Reprod Dev 2008;75:1617-1626.

20 Zhang DX, Park WJ, Sun SC, Xu YN, Li YH, Cui XS, Kim NH: Regulation of maternal gene expression by MEK/ MAPK and MPF signaling in porcine oocytes during in vitro meiotic maturation. J Reprod Dev 2011;57:4956.

21 Zhang Y, Sheets MD: Analyses of zebrafish and Xenopus oocyte maturation reveal conserved and diverged features of translational regulation of maternal cyclin B1 mRNA. BMC Dev Biol 2009;9:7.

22 Jang WI, Lin ZL, Lee SH, Namgoong S, Kim NH: A specific inhibitor of CDK1, RO-3306, reversibly arrests meiosis during in vitro maturation of porcine oocytes. Anim Reprod Sci 2014;144:102-108.

23 Reyes JM, Chitwood JL, Ross PJ: RNA-Seq profiling of single bovine oocyte transcript abundance and its modulation by cytoplasmic polyadenylation. Mol Reprod Dev 2015;82:103-114.

24 Peluso C, Fonseca FL, Gastaldo GG, Christofolini DM, Cordts EB, Barbosa CP, Bianco B: AMH and AMHR2 polymorphisms and AMH serum level can predict assisted reproduction outcomes: a cross-sectional study. Cell Physiol Biochem 2015;35:1401-1412. 